\title{
Estudio descriptivo sobre el uso de antiinflamatorios no esteroideos por vía intramuscular para el tratamiento de la lumbalgia aguda en las consultas de Atención Primaria en España durante 2002-2011
}

\author{
Rafael Sáez-Jiménez ${ }^{a}$ y Julio Bonis ${ }^{b}$
}

\begin{abstract}
a Médico especialista en Medicina Familiar y Comunitaria. Centro de Salud Presentación Sabio. Móstoles. Madrid (España).

${ }^{\mathrm{b}}$ Médico especialista en Medicina Familiar y Comunitaria. Programa BIFAP. División de Farmacoeconomía y Farmacovigilancia. Agencia Española del Medicamento y Productos Sanitarios. Madrid (España).
\end{abstract}

\section{Correspondencia:}

Rafael Sáez-Jiménez.

Centro de Salud Presentación Sabio.

C/ Alonso Cano, 8. C.P. 28933. Móstoles - Madrid (España).

Correo electrónico:

rsaezj@gmail.com

Recibido el 15 de abril de 2015. Aceptado para su publicación el 11 de mayo de 2015.

\section{RESUMEN}

Objetivo: Describir la evolución de la prescripción de antiinflamatorios no esteroideos (AINE) por vía intramuscular para el tratamiento de un primer episodio de lumbalgia en Atención Primaria en España entre 2002 y 2011.

Diseño del estudio: Estudio descriptivo transversal.

Emplazamiento: Atención Primaria.

Participantes: 604.423 pacientes $\geq 20$ años con al menos un episodio de lumbalgia registrado en la historia de la base de datos BIFAP.

Mediciones principales: Las variables estudiadas fueron los episodios codificados por L03, L84 y L86 según la Clasificación Internacional de Atención Primaria (CIAP-2) y los fármacos de los grupos M01A, N02BB02 y N02BB91 de la clasificación Anatómico-Terapéutica-Química de la OMS. También las variables sexo y edad.

Resultados: La edad media de diagnóstico de un primer episodio de lumbalgia fue de 49 años; el 59\% eran mujeres. Se prescribieron 342.514 AINE, el 95,51 \% por vía oral (el más prescrito ibuprofeno), el 4,11 \% por vía intramuscular (el más prescrito diclofenaco) y el 0,38 \% por vía rectal (el más prescrito diclofenaco). La prescripción por vía intramuscular se asoció a ser varón, lumbalgia irradiada y la edad.

Conclusiones: La prescripción de AINE para el tratamiento del dolor lumbar ha ido aumentando en el periodo estudiado. A pesar de que se observa una tendencia a la disminución, la vía intramuscular se sigue utilizando, por lo que sería recomendable incluir en las guías de práctica clínica recomendaciones acerca del uso de esta vía de administración de AINE para el tratamiento del dolor lumbar.

PALABRAS CLAVE: Lumbalgia. Antiinflamatorios no Esteroideos. Inyecciones Intramusculares. Atención Primaria de Salud.

\section{ABSTRACT}

Descriptive study on the use of NSAIDs intramuscularly for the treatment of acute low back pain in primary care consultations in Spain during 2002-2011

Objective: To describe the evolution of intramuscular non-steroidal anti-inflammatory (NSAID) prescriptions for the treatment of a first episode of low back pain in primary care in Spain between 2002 and 2011.

Study design: Descriptive cross-sectional study.

Setting: Primary care.

Participants: 604,423 patients $\geq 20$ years who had a first episode of low back pain registered on the BIFAP database.

Measurements: The variables studied were coded episodes L03, L84 and L86 according to the International Classification of Primary Care (ICPC-2) and the drugs M01A, N02BB02 and N02BB91 groups of Anatomical-Therapeutic-Chemical WHO classification. Also sex and age variables.

Results: The average age of diagnosis of a first episode of back pain was 49 years; $59 \%$ were women. 342,514 NSAID were prescribed, $95.51 \%$ orally (the most frequently prescribed being ibuprofen), $4.11 \%$ intramuscularly (the most frequently prescribed being diclofenac) and $0.38 \%$ rectally (the most frequently prescribed being diclofenac). Intramuscular prescription was associated with being male, radiated low back pain and age.

Conclusions: The prescription of NSAIDs for the treatment of low back pain has increased in the period studied. Although a downward trend is observed, the intramuscular route is still used. Therefore it would be advisable to include recommendations about the use of this route of administration of NSAID for the treatment of low back pain in clinical practice guidelines.

KEY WORDS: Low Back Pain. Antiinflammatory Agents, Non-Steroidal. Injections, Intramuscular. Primary Health Care. 


\section{INTRODUCCIÓN}

La lumbalgia representa un importante problema de salud pública en la sociedad occidental por su elevada prevalencia y repercusión socioeconómica: origina una elevada utilización de los servicios sanitarios, consultas a diferentes profesionales y una considerable pérdida de jornadas laborales ${ }^{1-3}$.

Una reciente revisión sistemática a nivel mundial estima una prevalencia del 9,4 \% ${ }^{1}$. A nivel nacional, refiriéndonos a la lumbalgia aguda el estudio EPISER ${ }^{4}$ obtiene una prevalencia del $14,8 \%$.

Su tratamiento es objetivo de numerosas guías y revisiones; entre esos tratamientos se encuentran los antiinflamatorios no esteroideos (AINE) ${ }^{5-9}$. Las guías aconsejan su utilización como segundo escalón tras el paracetamol, aunque según una revisión Cochrane ${ }^{10}$ existe limitada evidencia de que los AINE sean más efectivos que el paracetamol en el tratamiento de la lumbalgia crónica. Unido a sus potenciales efectos secundarios hace que los AINE deban utilizarse, en la medida que la patología de base lo permita, en ciclos cortos de tratamiento y a las dosis más bajas posibles, siempre dentro de su rango de eficacia, y vigilando de manera específica las complicaciones digestivas, cardiovasculares, renales, hepáticas y hematológicas.

Aunque las guías para el tratamiento de la lumbalgia a nivel local, nacional e internacional no mencionan la utilización de AINE por vía intramuscular, en las consultas de atención primaria se sigue utilizando para el alivio del dolor lumbar agudo. Los argumentos por parte del médico y las creencias de los pacientes presuponen la superioridad de la vía intramuscular, tanto en la intensidad como en la rapidez del efecto, apoyándose estos argumentos por un posible efecto placebo. Este efecto tampoco ha sido demostrado.

El objetivo del estudio es describir la evolución de la prescripción de AINE por vía intramuscular para el tratamiento de un primer episodio de lumbalgia en Atención Primaria en España, su evolución en la década 2002 al 2011 y los factores asociados a su uso.

\section{SUJETOS Y MÉTODOS}

Se trata de un estudio descriptivo transversal. La población del estudio comprende la incluida en la base de datos BIFAP versión 2011 (Base de da- tos para la investigación fármaco-epidemiológica en Atención Primaria) ${ }^{11}$. Es una base de datos informatizada de registros médicos en el ámbito de Atención Primaria, que incluye la información aportada por 2.324 médicos de Atención Primaria del Sistema de Salud de 10 Comunidades Autónomas de las 17 de toda España.

Se seleccionaron aquellos pacientes mayores de 20 años con información disponible en BIFAP que tenían algún episodio de lumbalgia registrado en su historia clínica durante el periodo 2002 a 2011. El análisis se realizó sobre el primer episodio de lumbalgia de cada paciente seleccionado. Para ello las variables estudiadas fueron los códigos L03 (lumbalgia sin irradiación), L84 (lumboartrosis, osteoartrosis de columna, artrosis degenerativa de columna) y L86 (lumbociática, síndrome discal lumbar) según la Clasificación Internacional de Atención Primaria (CIAP-2).

Para el análisis del uso de AINE las variables estudiadas fueron los registros de prescripciones de los fármacos del Grupo M01A (productos antiinflamatorios y antirreumáticos no esteroideos), N02BB02 (metamizol sódico) y N02BB91 (metamizol magnésico) de la clasificación Anatómico-TerapéuticaQuímica de la OMS, pautados en su formulación oral, rectal y parenteral prescritos en los 14 días posteriores al primer episodio de lumbalgia.

También se analizaron las variables sexo y edad.

La asociación entre variables se estudió con un modelo de regresión logística multivariante utilizando el software STATA versión 12. Las variables independientes para el modelo de regresión logística multivariante fueron: sexo, edad y lumbalgia con irradiación o no irradiada y fármaco utilizado, mientras que se consideró como variable dependiente la prescripción por vía intramuscular.

\section{RESULTADOS}

De las 2.571.015 historias clínicas analizadas, se registraron 604.423 episodios de lumbalgia, para cuyo tratamiento se prescribieron 342.514 AINE en los 14 días inmediatamente posteriores al episodio de lumbalgia. La edad media de diagnóstico fue de 49 años; el $59 \%$ eran mujeres.

De forma global, sin especificar la vía de administración, el porcentaje de prescripción pasa de un $48,51 \%$ en el año 2002 a un 56,24 \% en el año 
2011; ese aumento es mayor en 2007, donde se produce un pico $(59,20 \%)$. El principio activo más pautado fue el ibuprofeno seguido del diclofenaco.

Diferenciando la vía de administración, la evolución anual de la prescripción de las tres vías de administración está representada en el figura 1. El $95,51 \%$ de las prescripciones fueron por vía oral (el más prescrito ibuprofeno: $42,87 \%$ ), el $4,11 \%$ por vía intramuscular (el más prescrito diclofenaco: $50,62 \%$ ) y el $0,38 \%$ por vía rectal (el más prescrito diclofenaco: $73,52 \%$ ).

La evolución de la vía intramuscular y rectal está representada en la figura 2.

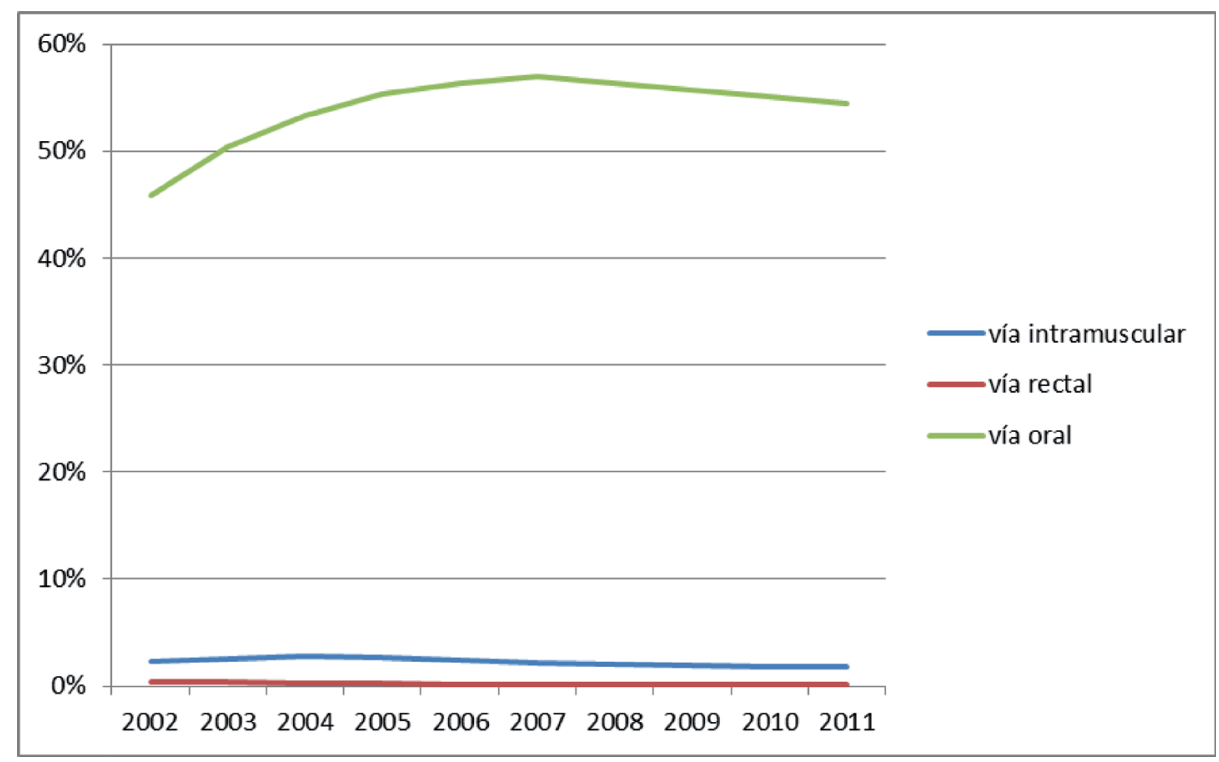

Figura 1. Evolución de la prescripción de AINEs para el tratamiento de un primer episodio de lumbalgia según las diferentes vías de administración

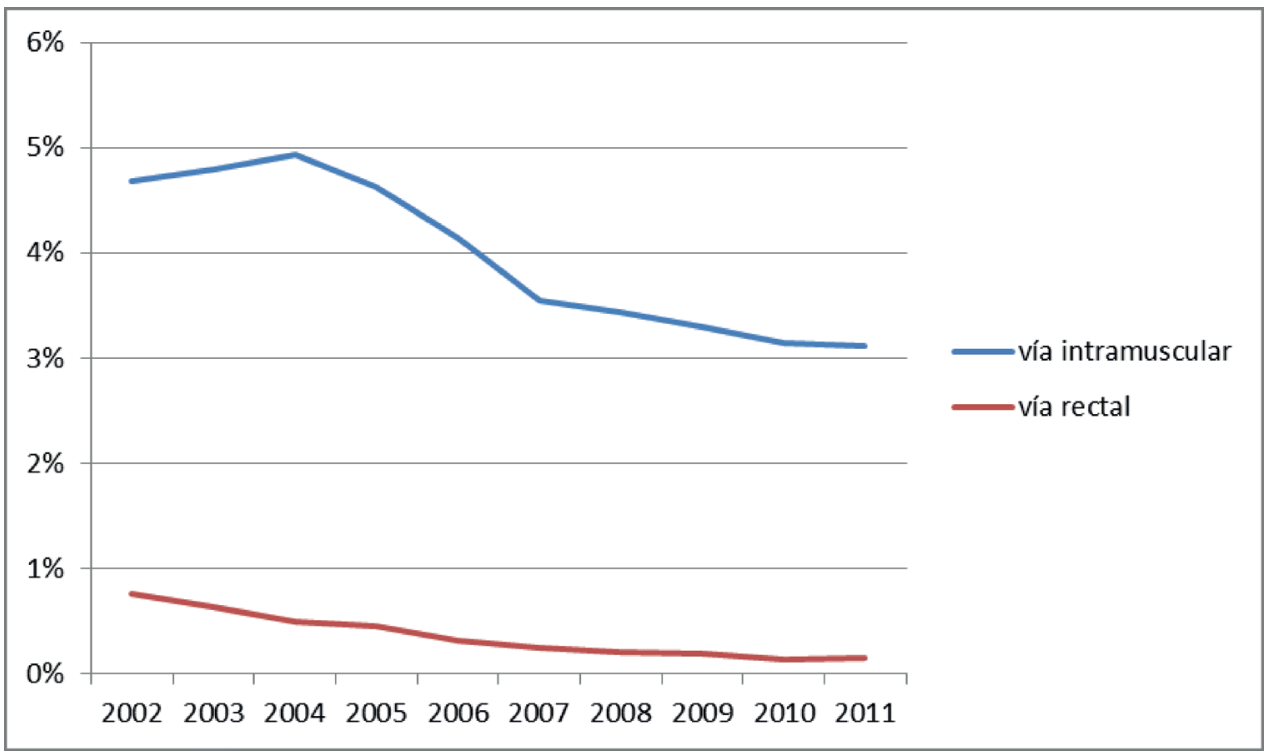

Figura 2. Evolución de la prescripción de AINEs para el tratamiento de un primer episodio de lumbalgia comparando la vía de administración intramuscular y rectal 
Atendiendo a la vía de administración intramuscular de AINE, el modelo de regresión logística encontró una asociación estadísticamente significativa entre la prescripción de AINE por vía intramuscular y el hecho de ser varón, tener un episodio de lumbalgia con irradiación y la edad (tabla 1).

\begin{tabular}{lcc}
\hline Variables & Odds ratio $^{1}$ & Intervalo de confianza 95 \% \\
\hline Varón & $\mathbf{1 , 0 5}$ & $\mathbf{1 , 0 1 - 1 , 0 8}$ \\
Edad & 1,00 & \\
20 a 20 años & 1,41 & \\
30 a 39 años & 1,91 & $1,30-1,54$ \\
40 a 49 años & 2,46 & $1,76-2,08$ \\
50 a 59 años & 2,84 & $2,26-2,67$ \\
60 a 69 años & 3,78 & $2,61-3,09$ \\
70 a 79 años & 4,38 & $3,47-4,12$ \\
80 o más años & 1,78 & $3,97-4,83$ \\
Dolor irradiado & & $1,72-1,85$ \\
\hline
\end{tabular}

Tabla 1. Modelo de regresión logística multivariante. ${ }^{1}$ Odds ratio de usar la vía intramuscular entre aquellos pacientes que recibieron un AINE en los 14 días posteriores a un primer episodio de lumbalgia. ${ }^{2}$ Episodios de lumbalgia codificados como "L86 Síndrome lumbar con irradiación”.

\section{DISCUSIÓN}

El aumento progresivo del consumo de AINE encontrado en nuestro estudio también es descrito por el Ministerio de Sanidad, Servicios Sociales e Igualdad $^{12}$. Ellos objetivan un aumento del $26,5 \%$ desde el año 2000 al 2012, aunque refieren que desde el 2009 se observa una tendencia hacia la disminución. Si observamos la figura 1, vemos que dicha tendencia ya se refleja en nuestro estudio a partir del año 2008, aunque tenemos que tener en cuenta que los datos del Ministerio se refieren a AINE consumidos para cualquier patología y nuestros resultados están centrados en la lumbalgia.

Refiriéndonos a la lumbalgia y a la vía de administración oral, según un estudio de Kovacs et al. ${ }^{13}$ el $57,1 \%$ de los pacientes con diagnóstico de lumbalgia no específica recibieron un AINE vía oral ya en la primera consulta del médico de atención primaria, coincidiendo con nuestro porcentaje $(56,6 \%)$.

No disponemos de ningún otro estudio que describa la evolución del consumo de AINE para la lumbalgia en función de la vía de administración.

En el figura 1 observamos que la gran mayoría de los AINE fueron prescritos por vía oral, pasando de un $45,86 \%$ en 2002 a un $54,40 \%$ en 2011 ; dado que es la vía más utilizada su evolución coincide con la evolución del consumo global por todas las vías.

El interés del estudio está en analizar la evolución de las otras dos vías minoritarias, especialmente la intramuscular; por este motivo y porque el porcentaje total de ambas vías en relación con la vía oral es menor (4,49\%), las hemos representado en un gráfico distinto (figura 2). Vemos que la vía de administración intramuscular tiene un pequeño ascenso desde el año 2002 al 2004, que supone un 4,69 y un $4,93 \%$ respectivamente, para después iniciar un descenso paulatino, más marcado hasta el año 2007 y a partir de aquí el descenso es más progresivo con un pequeño estancamiento en los dos últimos años, representando un 3,15 y un $3,11 \%$ respectivamente. La vía de administración rectal presenta un descenso desde el año 2002 $(0,76 \%)$ a un $0,15 \%$ en 2011.

El modelo de regresión logística (tabla 1) mostró una asociación entre la prescripción de AINE por vía intramuscular con las variables independientes sexo masculino, lumbalgia con irradiación y la edad. En el resto de variables como tipo de fármaco utilizado y lumbalgia no irradiada no se encontró asociación.

Referente al sexo discrepamos con el estudio 
EPISER ${ }^{4}$, pues observaron un mayor consumo de AINE en las mujeres; si bien este consumo fue para el tratamiento del dolor musculoesquelético en general, no solo para la lumbalgia, las diferencias encontradas no fueron estadísticamente significativas y la vía de administración era oral.

La asociación entre la vía intramuscular y la lumbalgia irradiada posiblemente sea debida a que estos pacientes, al presentar radiculopatías, tengan mayor discapacidad funcional lo que pudiera influir en prescribir una vía más agresiva.

La edad ${ }^{14}$ también se asoció a la utilización de la vía intramuscular, de tal forma que los pacientes de mayor edad recibieron con más frecuencia AINE vía intramuscular; este incremento ya es significativo a partir de los 40 años. La edad es el factor con una mayor magnitud de efecto.

Los efectos secundarios de los AINE son unos de los motivos por los que las guías de lumbalgia los recomiendan como segundo escalón. A estos se añaden los efectos inherentes a la vía de administración, es el caso de la vía intramuscular (necrosis, abscesos, fascitis). Hemos revisado la Base de datos Europea de informes de presuntas reacciones adversas ${ }^{15}$ sin encontrar ninguna notificación al respecto. Estos efectos aparecen aisladamente en la literatura médica como casos clínicos ${ }^{16-24}$.

En los estudios ${ }^{25-29}$ que comparan la eficacia y los efectos secundarios de los AINE administrados por vía oral versus vía intramuscular para tratar el dolor agudo musculoesquelético, se concluye que no es más eficaz la vía intramuscular. Las revisiones sistemáticas ${ }^{30,31}$ indican que la vía intramuscular podría estar justificada para el tratamiento del dolor agudo de procesos que se acompañan de náuseas y vómitos, como puede ser en el cólico renal; en los demás casos hace falta más evidencia.

La relación médico-paciente en estas situaciones es fundamental; es importante informar a los pacientes de los riesgos asociados y consensuar con ellos la vía de administración, pues la predisposición del paciente influye en el efecto del tratamiento ${ }^{14,29,32}$.

Este estudio pone de manifiesto el uso de AINE por vía intramuscular para el tratamiento de la lumbalgia; aunque el porcentaje no es excesivo, de un $4,11 \%$ en el periodo estudiado, la elevada prevalencia de la lumbalgia en la población $n^{1,4}$ lo convierte en un problema potencial en términos globales.

La indicación de la vía de administración intramuscular no se recoge en las guías clínicas revisadas por los autores. Esta ausencia de información podría interpretarse como que no se recomienda su uso; aunque nuestros datos muestran que sí es utilizada en las consultas de Atención Primaria. Creemos por tanto conveniente que las guías de práctica clínica incluyan recomendaciones sobre esta vía de administración en uno u otro sentido.

Entre las posibles limitaciones del estudio está el que el número de prescripciones de AINE no tiene por qué ser un reflejo exacto de su utilización real, ya que el médico puede haber prescrito un AINE intramuscular pero éste no ser dispensado o administrado finalmente. $Y$ al contrario, puede que no exista una prescripción registrada, pero se administre el fármaco del stock que cada centro de salud cuenta en su botiquín. Adicionalmente, de la base de datos extraemos prescripciones parenterales, que aparte de la vía intramuscular también incluye la vía intravenosa, aunque esta última vía es más excepcional en primaria y más propia de atención hospitalaria. Pero en estudios con amplia muestra poblacional es difícil conocer el grado de cumplimiento de los pacientes; así el propio estudio de consumo de AINE del Ministerio de Sanidad, Servicios Sociales e Igualdad" indica que "se ha utilizado la información sobre facturación de recetas del Sistema Nacional de Salud", que también es una medida indirecta del consumo.

\section{AGRADECIMIENTOS}

Agradecemos a los médicos de atención primaria sin cuya colaboración con el programa BIFAP este estudio no habría sido posible.

El autor manifiesta la inexistencia de conflicto de intereses en la realización del estudio, que se ha llevado a cabo sin financiación externa y con independencia de la industria farmacéutica. 


\section{BIBLIOGRAFÍA}

1. Hoy D, March L, Brooks P, Blyth F, Woolf A, Bain C et al. The global burden of low back pain: estimates from the Global Burden of Disease 2010 study. Ann Rheum Dis. 2014; 73 (6): 968-74.

2. Dagenais S, Caro J, Haldeman S. A systematic review of low back pain cost of illness studies in the United States and internationally. Spine J. 2008; 8 (1): 8-20.

3. Maniadakis N, Gray A. The economic burden of back pain in the UK. Pain. 2000; 84 (1): 95-103.

4. Humbría Mendiola A, Carmona L, Peña Sagredo JL, Ortiz AM. Impacto poblacional del dolor lumbar en España: resultados del estudio EPISER. Rev Esp Reumatol. 2002; 29 (10): 471-8.

5. Grupo Español del Programa Europeo COST B13. Guía de Práctica Clínica para la Lumbalgia Inespecífica 2005. Disponible en: http://www.reide.org [consultado 4 de mayo de 2015].

6. Koes BW, van Tulder MW, Thomas S. Diagnosis and treatment of low back pain. BMJ. 2006; 332 (7555): 1430-4.

7. van Tulder M, Becker A, Bekkering T, Breen A, del Real MT, Hutchinson A et al; COST B13 Working Group on Guidelines for the management of acute low back pain in primary care. Chapter 3. European guidelines for the management of acute nonspecific low back pain in primary care. Eur Spine J. 2006; 15 Suppl 2: S169-91.

8. Airaksinen O, Brox JI, Cedraschi C, Hildebrandt J, KlaberMoffett J. Kovacs F et al; COST B13 Working Group on Guidelines for chronic low back pain. Chapter 4. European guidelines for the management of chronic nonspecific low back pain. Eur Spine J. 2006; 15 Suppl 2: S192-300.

9. Pérez Irazusta I, Alcorta Michelena I, Aguirre Lejarcegui G, Aristegi Racero G, Caso Martínez J, Esquisabel Martínez R et al. Guía de Práctica Clínica sobre Lumbalgia Osakidetza. GPC 2007/1. Vitoria-Gasteiz; 2007.

10. Roelofs PD, Deyo RA, Koes BW, Scholten RJ, van Tulder MW. Non-steroidal anti-inflammatory drugs for low back pain: an updated Cochrane review. Spine 2008; 33 (16): 1766-74.

11. Salvador Rosa A, Moreno Pérez JC, Sonego D, García Rodríguez LA, de Abajo Iglesias FJ. El Proyecto BIFAP: Base de datos para la Investigación Fármacoepidemiológica en Atención Primaria. Aten Primaria. 2002; 30 (10): 655-61.

12. Ministerio de Sanidad, Servicios Sociales e Igualdad. Agencia Española de Medicamentos y Productos Sanitarios. Utilización de medicamentos antiinflamatorios no esteroideos (AINE) en España durante el periodo 20002012. Informe de Utilización de Medicamentos U/AIN/ V1/15012014. Disponible en: http://www.aemps.gob.es/ medicamentosUsoHumano/observatorio/docs/AINE.pdf [publicado el 27 de enero de 2014; consultado el 4 de mayo 2015)

13. Kovacs FM, Fernández C, Cordero A, Muriel A, GonzálezLuján L, Gil del Real MT; Spanish Back Pain Research
Network. Non-specific low back pain in primary care in the Spanish National Health Service: a prospective study on clinical outcomes and determinants of management. BMC Health Serv Res. 2006; 6: 57.

14. González González J, Berrocoso Martínez A, de Arriba Muñoz L, Rico García G, Izaguirre Zapatera A, Alfonso Izquierdo MJ. El arte de curar: estudio sobre vías de administración. Diferencias entre medio rural y urbano. Rev Clin Med Fam. 2009; 2 (7): 340-3.

15. Eudravigilance [Internet]. Disponible en: http://www.eudravigilance.ema.europa.eu/human/index.asp [consultado 4 de mayo 2015]

16. Serratrice G. Study of diclofenac injectable. Tribune Med. 1982; 46: 43-8.

17. Rygnestad T, Kvam AM. Streptococcal myositis and tissue necrosis with intramuscular administration of diclofenac (Voltaren). Acta Anaesthesiol Scand. 1995; 39 (8):1128-30.

18. Pillans $\mathrm{PI}, \mathrm{O}$ 'Connor $\mathrm{N}$ : Tissue necrosis and necrotizing fasciitis after intramuscular administration of diclofenac. Ann Pharmacother. 1995; 29 (3): 264-6.

19. Schäbitz WR, Berger $C$, Knauth $M$, Meinck HM, Steiner $T$. Hypoxic brain damage after intramuscular self-injection of diclofenac for acute back pain. Eur J Anaesthesiol. 2001; 18 (11): 763-5.

20. Frick S, Cerny A. Necrotizing fasciitis due to Streptococcus pneumoniae after intramuscular injection of nonsteroidal anti-inflammatory drugs: report of 2 cases and review. Clin Infect Dis. 2001; 33 (5): 740-4.

21. McGee AM, Davison PM. Skin necrosis following injection of non-steroidal anti-inflammatory drug. $\mathrm{Br} \mathrm{J}$ Anaesth. 2002; 88 (1): 139-40.

22. Verfaillie G, Knape S, Corne L. A case of fatal necrotizing fasciitis after intramuscular administration of diclofenac. Eur J Emerg Med. 2002; 9 (3):270-3.

23. Okan G, Yaylaci $S$, Ince $U$, Zorer S: Necrotizing fasciitis following intramuscular diclofenac injection. J Eur Acad Dermatol Venereol. 2008; 22 (12): 1521-2.

24. Velissaris D, Matzaroglou C, Kalogeropoulou C, Karamouzos V, Filos K and Karanikolas M. Sepsis requiring intensive care following intramuscular injections: two case reports. Cases J. 2009; 2:7365.

25. Turturro MA, Paris PM, Seaberg DC. Intramuscular ketorolac versus oral ibuprofen in acute musculoskeletal pain. Ann Emerg Med. 1995; 26 (2): 117-20.

26. Auvinet $B$, Ziller R, Appelboom $T$, Velicitat $P$. Comparison of the onset and intensity of action of intramuscular meloxicam and oral meloxicam in patients with acute sciatica. Clin Ther. 1995; 17 (6): 1078-98.

27. Colberg K, Hettich M, Sigmund R, Degner FL. The efficacy and tolerability of an 8-day administration of intravenous and oral meloxicam: a comparison with intramuscular and oral diclofenac in patients with acute lumbago. German Meloxicam Ampoule Study Group. Curr Med Res Opin. 1996; 13 (7): 363-77.

28. Bosch HC, Sigmund R, Hettich M. Efficacy and tolerability 
of intramuscular and oral meloxicam in patients with acute lumbago: a comparison with intramuscular and oral piroxicam. Curr Med Res Opin. 1997; 14 (1): 29-38.

29. Schwartz NA, Turturro MA, Istvan DJ, Larkin GL. Patients' perceptions of route of nonsteroidal anti-inflammatory drug administration and its effect on analgesia. Acad Emerg Med. 2000; 7 (8): 857-61.

30. Tramèr MR, Williams JE, Carroll D, Wiffen PJ, Moore RA, McQuay HJ. Comparing analgesic efficacy of non-steroidal anti-inflammatory drugs given by different routes in acu- te and chronic pain: a qualitative systematic review. Acta Anaesthesiol Scand. 1998; 42 (1): 71-9.

31. Arora S, Wagner JG, Herbert M. Myth: parenteral ketorolac provides more effective analgesia than oral ibuprofen. CJEM. 2007; 9 (1): 30-2.

32. Rosemann $T$, Joos $S$, Koerner $T$, Heiderhoff M, Laux G, Szecsenyi J. Use of a patient information leaflet to influence patient decisions regarding mode of administration of NSAID medications in case of acute low back pain. Eur Spine J .2006; 15 (11): 1737-41 